\title{
Retinopexia pneumática e fotocooagulação a laser para tratamento de descolamento secundário à fosseta de disco óptico: relato de caso
}

\author{
Pneumatic retinopexy and laser photocoagulation for treatment of optic disc pit \\ detachment:case report
}

\author{
Alexandre Antonio Marques Rosa ${ }^{1}$ \\ Hélio Paulo Primiano Jr.2 \\ Yoshitaka Nakashima ${ }^{3}$
}

Trabalho realizado na Clínica Oftalmológica do Hospital de Clinicas da Faculdade de Medicina da Universidade de São Paulo - USP - São Paulo (SP) - Brasil.

${ }^{1}$ Pós-graduando nível doutorado da Faculdade de Medicina da Universidade de São Paulo - USP - São Paulo (SP). Professor da Disciplina de Oftalmologia da Universidade Federal do Pará - UFPA - Belém (PA) - Brasil. Médico da Clínica Oftalmológica do Hospital das Clínicas da Faculdade de Medicina da USP - São Paulo (SP) - Brasil.

${ }^{3}$ Médico Assistente do Setor de Retina e Vítreo da Clínica Oftalmológica do Hospital das Clínicas da Faculdade de Medicina da USP - São Paulo (SP) - Brasil.

Endereço para correspondência: Alexandre Antonio Marques Rosa. Trav. Lomas Valentinas, 1897/402 Belém (PA) CEP 66087-440

E-mail: alexandre_ros@hotmail.com

Recebido para publicação em 05.05.2005

Versão revisada recebida em 27.09.2005

Aprovação em 25.10.2005

Nota Editorial: Depois de concluída a análise do artigo sob sigilo editorial e com a anuência do Dr. Ayrton Roberto Branco Ramos sobre a divulgação de seu nome como revisor, agradecemos sua participação neste processo.

\section{RESUMO}

Os autores relatam o caso de um paciente com fosseta congênita de disco óptico associado a descolamento seroso macular, tratado com sucesso por meio de fotocoagulação com laser de argônio na borda temporal da fosseta, seguido de injeção intravítrea de $0,3 \mathrm{ml}$ de $\mathrm{C}_{3} \mathrm{~F}_{8}(100 \%)$. O paciente foi seguido por 12 meses. A resolução do descolamento foi acompanhada de melhora da acuidade visual, bem como o retorno da fóvea a sua configuração normal, comprovada através da tomografia de coerência óptica.

Descritores: Disco óptico/anormalidades; Doenças do nervo óptico/congênito; Processamento de imagem assistida por computador/métodos; Descolamento retiniano/etiologia; Coagulação por laser; Mácula lútea; Relatos de casos [tipo de publicação]

\section{INTRODUÇ̃̃OO}

A fosseta congênita do disco óptico é uma anormalidade rara, ocorrendo em aproximadamente 1:11.000 pacientes $^{(1)}$. É, geralmente, única e unilateral, freqüentemente associada a um disco óptico de tamanho aumentado e de borda irregular. Tipicamente, está localizada no setor temporal do disco óptico, embora possa estar em qualquer localização ${ }^{(2-3)}$.

A acuidade visual é boa e permanece inalterada, a menos que ocorra um descolamento seroso da mácula secundariamente ${ }^{(2)}$, o qual acontece em $25-75 \%$ dos $\operatorname{casos}^{(1,4-5)}$. O descolamento caracteristicamente se estende do disco óptico, nas vizinhanças da fosseta, e geralmente tem a forma de uma gota, com a parte mais estreita próxima ao disco óptico e se alargando em direção à mácula. A explicação fisiopatológica para esta complicação permanece controversa. Vazamento a partir de vasos na base da fosseta ${ }^{(6)}$, movimento de líquido do vítreo para o espaço sub-retiniano ${ }^{(4,7)}$ e fluxo de líquido cefalorraquidiano do espaço subaracnóideo para o espaço subretiniano $^{(5)}$ têm sido propostos.

Ao exame histopatológico, a fosseta consiste em uma depressão ou herniação do tecido neuroectodérmico rudimentar que contém elementos da retina sensorial, epitélio pigmentado da retina e tecido glial, que penetram pelo defeito do nervo óptico em direção ao espaço subaracnóideo perióptico $^{(1)}$.

A tomografia de coerência óptica (StratusOCT ${ }^{\circledR}$, Zeiss-Humphrey) é um método de imagem não invasivo e de não-contato capaz de produzir imagens através de cortes seccionais das estruturas oculares in vivo com uma 
resolução de 10 a $15 \mu \mathrm{m}^{(8)}$. É comparável, quanto à sua execução, ao ultra-som modo B, exceto por utilizar luz, ao invés de ondas sonoras, para a análise dos tecidos oculares. Por se tratar de um método digital, medidas quantitativas precisas podem ser obtidas. A tomografia de coerência óptica é um instrumento valioso no estudo das alterações maculares associadas às fossetas congênitas de disco óptico ${ }^{(9)}$.

Os autores trataram, através de fotocoagulação a laser de argônio seguido da injeção de $0,3 \mathrm{ml}$ de gás $\mathrm{C}_{3} \mathrm{~F}_{8}$ (100\%), um caso de descolamento seroso da região macular secundário à fosseta congênita do disco óptico.

\section{RELATO DE CASO}

MLM, 33 anos, feminino, parda, encaminhada com diagnóstico de buraco de mácula e baixa de visão no olho direito há um ano. A acuidade visual com a melhor correção era de 20/100 e 20/20, respectivamente no olho direito e esquerdo. A biomicroscopia, motilidade ocular extrínseca, reflexos pupilares e pressão intra-ocular eram normais. $\mathrm{O}$ exame de fundo do olho direito (Figura 1) mostrava uma fosseta congênita na região temporal do disco óptico com descolamento exsudativo no pólo posterior, buraco de mácula lamelar e uma artéria cilioretinana. A fundoscopia do olho esquerdo era normal.

Na tomografia de coerência óptica do olho direito, verificou-se um descolamento da retina neurossensorial com a formação de cistos intra-retinianos, correspondentes às áreas de retinosquise. Na região macular, observou-se a presença de um buraco lamelar.

Foi realizada fotocoagulação na borda temporal da fosseta (Figura 2) com mira de $100 \mu$, e potência suficiente para obter marcas de intensidade leve a moderada. Logo após foi realizada a injeção de $0,3 \mathrm{ml}$ de $\mathrm{C}_{3} \mathrm{~F}_{8}(100 \%)$, e orientado o paciente a ficar 10 dias ( 8 horas/dia) com a face voltada para baixo.

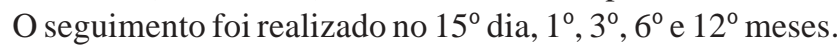
A tomografia de coerência óptica (StratusOCT ${ }^{\circledR}$, Zeiss-Humphrey) foi feita antes do tratamento e a cada retorno do paciente. Foram feitos seis tomogramas passando pela região macular, para análise da espessura macular no mapa retiniano.

O exame de tomografia de coerência óptica (Figura 3) demonstrou aplanamento progressivo do descolamento seroso macular, com restabelecimento da depressão foveal e melhora da acuidade visual, atingindo $20 / 25$ no $12^{\circ}$ mês (Figura 4 ).

\section{DISCUSSÃO}

As fossetas congênitas do disco óptico podem causar descolamento de retina e diminuição da acuidade visual. A localização e o tamanho da fosseta são importantes fatores predisponentes para o desenvolvimento de maculopatia. Fossetas grandes e localizadas temporalmente possuem maior $\operatorname{risco}^{(2-3)}$.
A origem do líquido sub-retiniano permanece controversa. Vazamento de líquido dos vasos da base da fosseta ${ }^{(6)}$, vítreo liquefeito $^{(4,7)}$ líquido cefalorraquidiano ${ }^{(5)}$ foram sugeridos como possíveis fontes.

Vazamento a partir de vasos é improvável, pois a angiografia fluorescente revela hipofluorescência precoce da fosseta e, em muitos casos, impregnação $\operatorname{tardia}^{(3)}$ sem extensão do corante para o espaço sub-retiniano.

Estudos de angiografia com indocianina verde demonstram um certo grau de hiperfluorescência tardia na região de descolamento macular, provavelmente devido ao vazamento do corante no espaço sub-retiniano ${ }^{(10)}$.

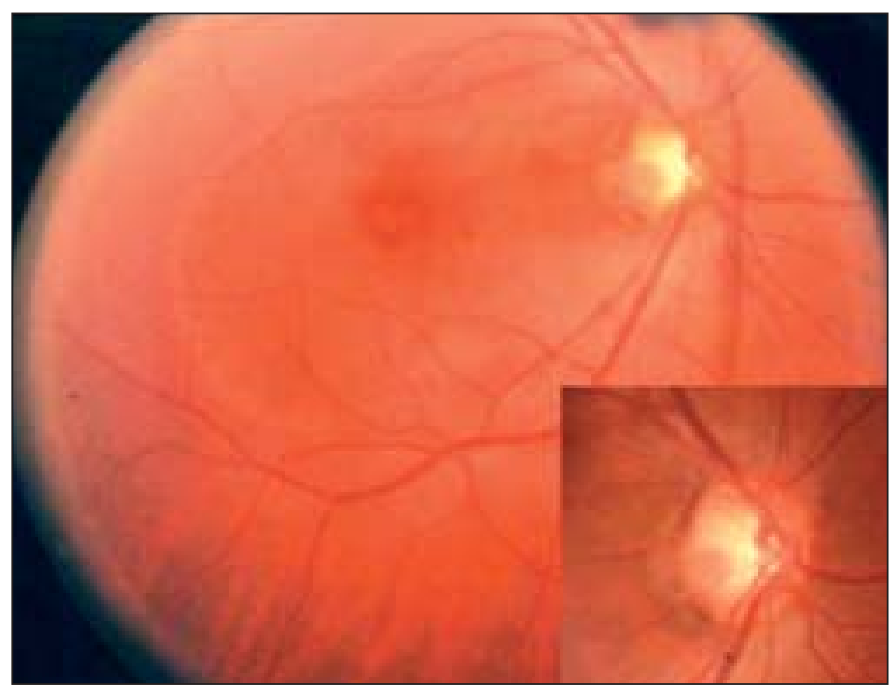

Figura 1 - Retinografia do olho direito onde se observa uma fosseta congênita de disco óptico na borda temporal, associada ao descolamento seroso macular. Quadro inferior direito mostrando a lesão em maior aumento

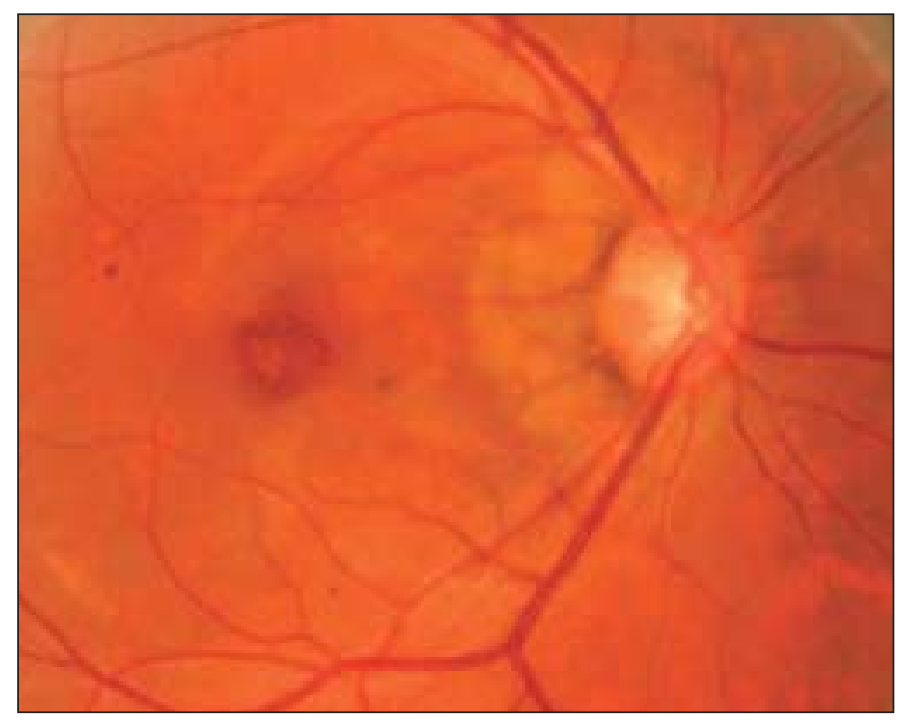

Figura 2 - Retinografia do olho direito imediatamente após a realização de três fileiras de fotocoagulação a laser na borda temporal do disco óptico 

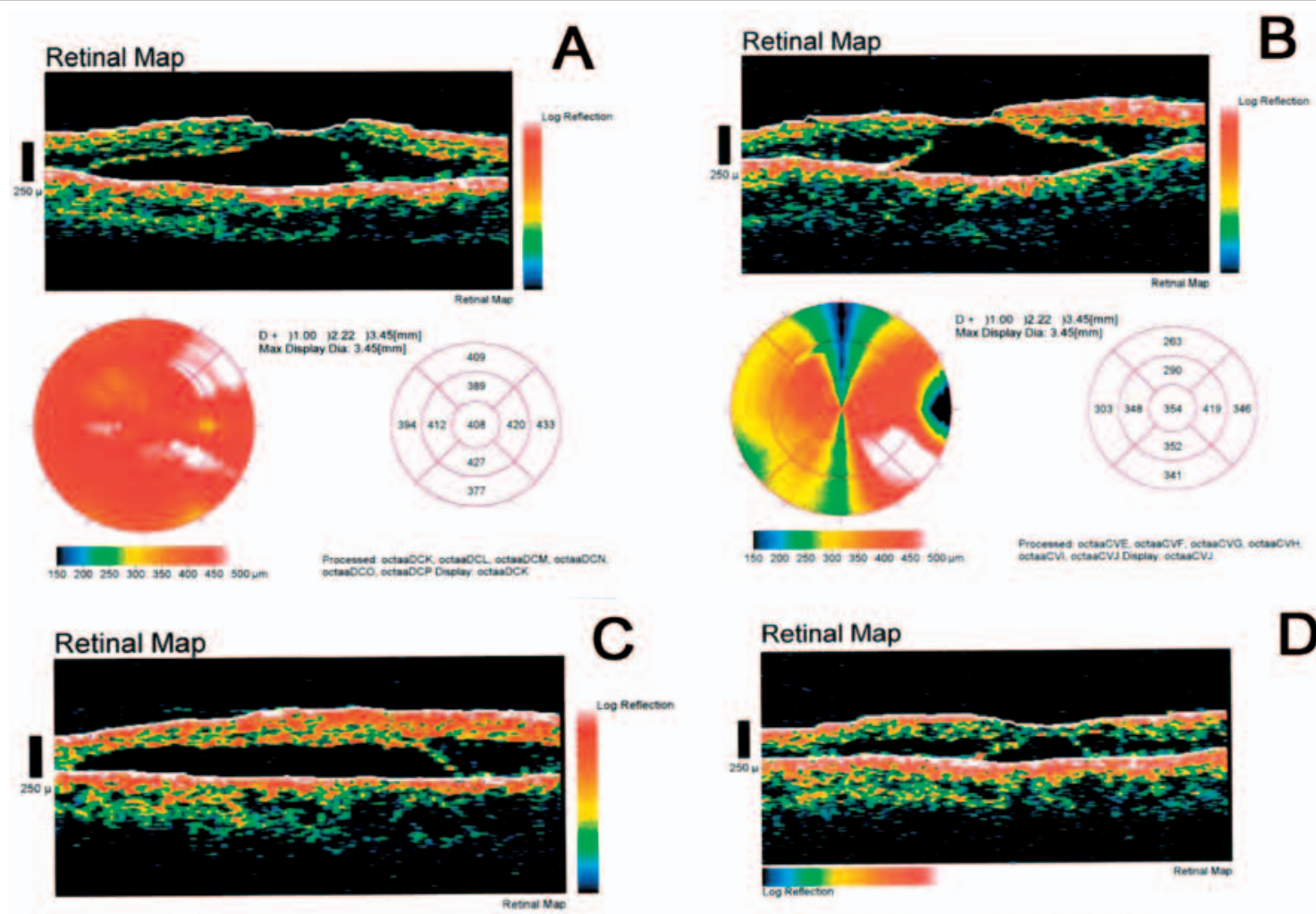

D

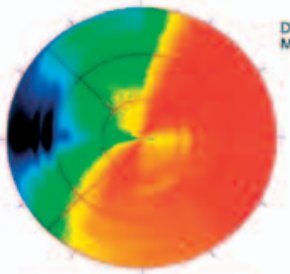

$150 \quad 200250900 \quad 950 \quad 400 \quad 450 \quad 500 \mathrm{um}$

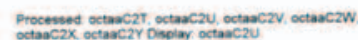

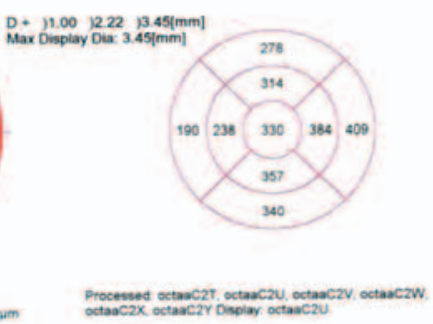

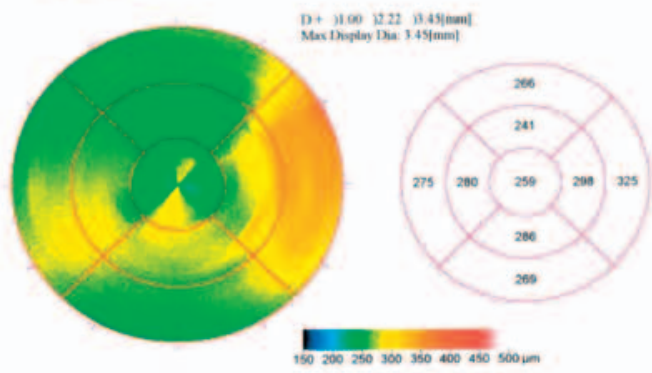
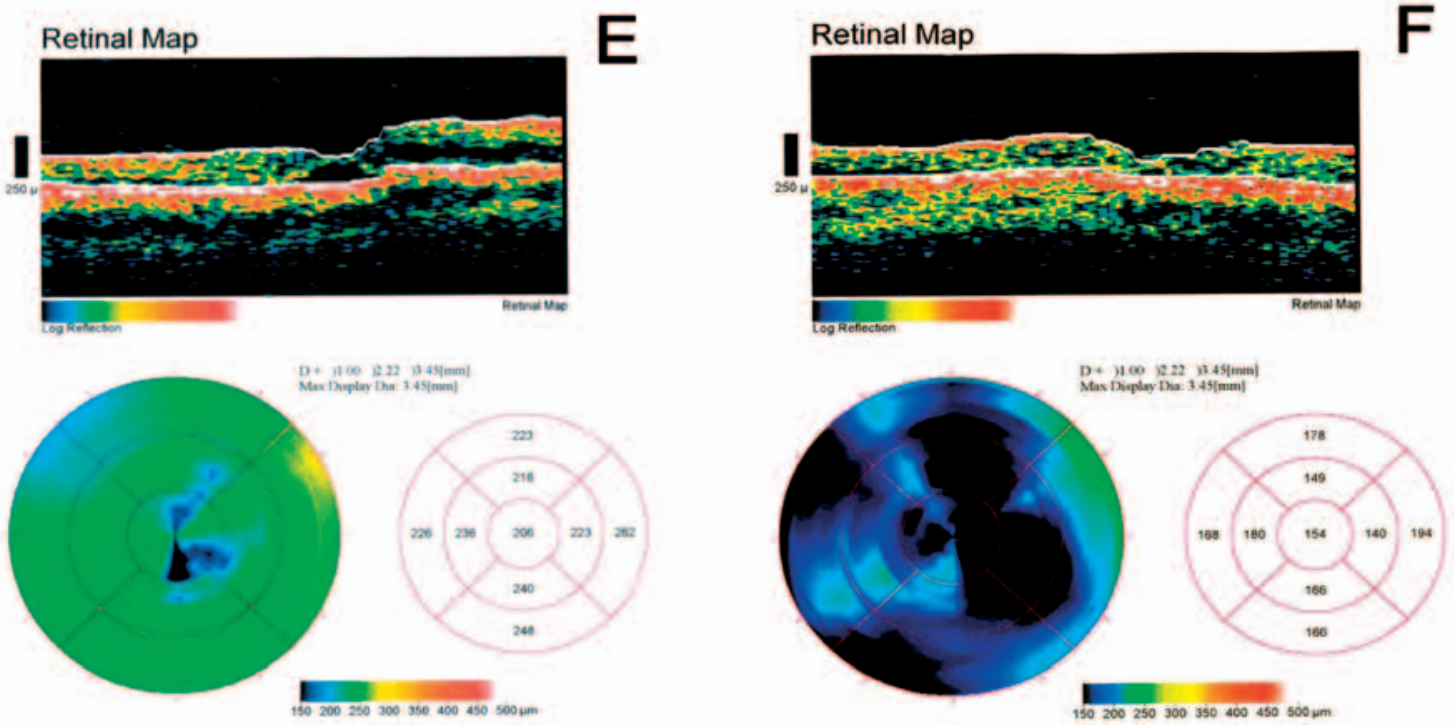

Figura 3 - Tomografia de coerência óptica (estratégia de análise: mapa retiniano) realizada no pré-operatório (A), 15 dias (B), 1 mês (C), 3 meses (D), 6 meses (E) e 12 meses (F) após o tratamento 


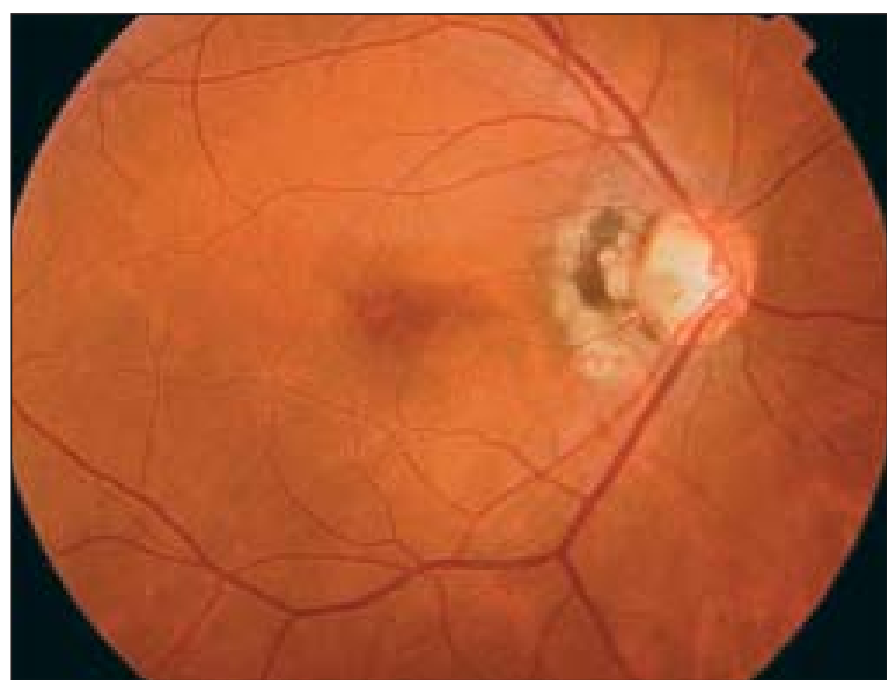

Figura 4 - Retinografia de olho direito mostrando as marcas da fotocoagulação a laser na borda do disco óptico e a resolução do descolamento seroso macular

Em modelo animal ${ }^{(7)}$, foi demonstrada a passagem de corante da cavidade vítrea para o espaço sub-retiniano, porém não foi identificado glicosaminoglicanos, um importante componente do vítreo, no espaço sub-retiniano. Além disso, os descolamentos maculares relacionados às fossetas de disco óptico ocorrem em pacientes muito jovens, os quais não costumam ter uma importante liquefação vítrea, tornando a hipótese do líquido ser proveniente do vítreo, pouco provável ${ }^{(2)}$.

Histologicamente, as fossetas são defeitos na lâmina crivosa e contém uma variedade de tecidos retinianos, incluindo nervos aberrantes e tecidos pigmentados, assemelhando-se ao epitélio pigmentado da retina, apoiados por uma rede de tecido glial $^{(1)}$. Histopatologicamente, existem bolsões contendo líquido que se estendem até as proximidades do espaço subaracnóide, mas não se comunicando com o mesmo ${ }^{(11)}$ apenas um fino septo separa estas estruturas do espaço sub-retiniano ${ }^{(5)}$.

Existe uma associação entre a fosseta de disco óptico e a separação semelhante à esquise que ocorre nas camadas internas da retina ${ }^{(12)}$. O fluído da fosseta eleva a camada de fibras nervosas, causando a separação das camadas retinianas internas. A esquise nas camadas internas progredirá para um descolamento retiniano seroso através de uma comunicação com a retina externa ${ }^{(12)}$.

A tomografia de coerência óptica demonstra evidências de retinosquise ou alterações císticas, com ou sem descolamento seroso da retina, em pacientes com fosseta congênita do disco óptico ${ }^{(9)}$.

A existência de pontes de fibras retinianas foram propostas para explicar a razão pela qual não se forma um escotoma absoluto nas regiões correspondentes às áreas de retinosquise ${ }^{(13)}$.

Tentativas de selar o descolamento na margem da fosseta pela fotocoagulação com laser têm sido mal sucedidas no que tange à resolução do descolamento macular ${ }^{(5,14)}$. A resolução pode ocorrer meses após o tratamento com fotocoagula- ção ${ }^{(15)}$. No entanto, recidiva do descolamento pode ocorrer nas semanas ou meses subseqüentes ${ }^{(16-17)}$. A ausência de separação do vítreo posterior, em pacientes com descolamento macular, sugere que a tração vítrea exercida nesta região pode desempenhar um importante papel na gênese do movimento passivo do fluído para o espaço sub-retiniano ${ }^{(16-19)}$.

Alguns autores relataram uma reaplicação bem sucedida da mácula após vitrectomia posterior via pars plana, endofotocoagulação e/ou tamponamento com gás ${ }^{(16-19)}$.

É possível que a injeção do gás, além de exercer um efeito tamponante, possa ter induzido o descolamento do vítreo posterior, liberando as trações que existiam sobre a região do descolamento, permitindo a reaplicação da retina após ter sido formada a adesão no local da fotocoagulação a laser. Duas possíveis explicações para a demora na reabsorção do líquido sub-retiniano seriam: tratar-se de um descolamento crônico, ou ainda pela diferente composição (osmolaridade) do líquido subretiniano. Ambas causariam uma dificuldade na capacidade de bombeamento das células do epitélio pigmentado da retina.

Em nosso país, existe grande dificuldade de acesso para pacientes do sistema público de saúde às cirurgias vítreoretinianas, portanto, esta modalidade de tratamento fornece grandes atrativos por ser de baixo custo e fácil execução. No entanto, estudos prospectivos adicionais, com período de acompanhamento longo, nos fornecerão um entendimento mais completo do mecanismo do descolamento da retina desta doença, bem como qual a melhor forma de tratamento.

\section{ABSTR ACT}

The authors report a case of congenital optic disc pit with serous macular detachment successfully treated, with argon laser photocoagulation in the temporal border of the pit, followed by intravitreous injection of $\mathrm{C}_{3} \mathrm{~F}_{8}(100 \%) 0.3 \mathrm{ml}$. Patient was followed up for 12 months. Retinal reattachment was associated with improvement in visual acuity, as well as return of the fovea to its normal configuration shown by optical coherence tomography.

Keywords: Optic disk/abnormalities; Optic nerve diseases/ congenital; Image processing, computer-assisted/methods; Retinal detachment/etiology; Laser coagulation; Macula lutea; Case reports [publications type]

\section{REFERÊNCIAS}

1. Kranenburg EW. Crater-like holes in the optic disc and central serous retinopathy. Arch Ophthalmol. 1960;64:912-24.

2. Brodsky MC. Congenital optic disk anomalies. Surv Ophthalmol. 1994;39(2): 89-112. Erratum in: Surv Ophthalmol. 1995;40(2):172.

3. Brown GC, Shields JA, Goldberg RE. Congenital pits of the optic nerve head: II. Clinical studies in humans. Ophthalmology. 1980;87(1):51-65.

4. Sugar HS. Congenital pits in the optic disk pit with acquired macular pathology. Am J Ophthalmol. 1962;53:307-11.

5. Gass JDM. Serous detachment of the macula secondary to congenital pit of the optic nerve head. Am J Ophthalmol. 1969;67(6):821-41. 
6. Gordon R, Chatfield RK. Pits in the optic disc associated with macular degeneration. Br J Ophthalmol. 1969;53(7):481-9.

7. Brown GC, Shields JA, Patty BE, Goldberg RE. Congenital pits of the optic nerve head I: experimental studies in collie dogs. Arch Ophthalmol. 1979;97(7): 1341-44.

8. Hee MR, Izatt JA, Swanson JA, Huang D, Schuman JS, Lin CP, et al. Optical coherence tomography of the human retina. Arch Ophthalmol. 1995;113(3):325-32.

9. Rosa AAM, Nakashima Y, Souza EC de. Aspectos à tomografia de coerência óptica de fosseta congênita de nervo óptico: relato de 3 casos. Arq Bras Oftalmol. 2002;65(3):369-73.

10. Theodossiadis GP, Ladas ID, Panagiotidis DN, Kollia AC, Voudouri AN, Theodossiadis PG. Fluorescein and indocyanine green angiographic findings in congenital optic disk pit associated with macular detachment. Retina. 1999;19(1):6-11.

11. Ferry AP. Macular detachment associated with congenital pit of the optic nerve head. Pathologic findings in two cases simulating malignant melanoma of the choroids. Arch Ophthalmol. 1963;70:346-57.

12. Lincoff H, Lopez R, Kreissig I, Yannuzzi L, Cox M, Burton T. Retinoschisis associated with optic nerve pits. Arch Ophthalmol. 1988;106(1):61-7.
13. Lincoff H, Yanuzzi L, Singerman L, Kreissig I, Fisher Y. Improvement in visual function at displacement of the retinal elevations emanating from optic pits. Arch Ophthalmol. 1993;111(8):1071-9.

14. Sugar HS. Congenital pits of the optic disk and their equivalents (congenital coloboma and coloboma-like excavations) associated with submacular fluid. Am J Ophthalmol. 1967;63(2):298-307.

15. Theodossiadis GP. Evolution of congenital pit of the optic disk with macular detachment in photocoagulated and nonphotocoagulated eyes. Am J Ophthalmol. 1977;84:620-31.

16. Schatz H, McDonald HR. Treatment of sensory retinal detachment associated with optic nerve pit or coloboma. Ophthalmology. 1988;95(2):178-86.

17. Zinn KM. Bilateral complete colobomas with a unilateral optic pit and recurrent retinal detachment: case report. Mt Sinai J Méd. 1979;46(4):419-23.

18. Snead MP, James N, Jacobs PM. Vitrectomy, argon laser, and gas tamponade for serous retinal detachment associated with an optic disc pit: a case report. $\mathrm{Br}$ J Ophthalmol. 1991;75(6):381-2.

19. Krasnik V, Strmen P, Hasa J, Izakova A, Hrachovcova J. [Surgical treatment of macular hole and maculopathy associated with optic disk pits]. Cesk Slov Oftalmol. 1999;55(5):263-7. Slovak.

\title{
IX Congresso Intemacional de Catarata e Cirurgia Refrativa
}

V Congresso Intemacional da Sociedade Brasileira de Administração em Oftalmologia

\section{1 de Maio a 03 de Junho de 2006 BELO HORIZONTE - MG}

\section{Informações sobre o evento da SBAO:}

\author{
Tel.: (21) 2285-6052 \\ Email: sbao@sbao.com.br \\ Home-page: www.sbao.com.br
}

